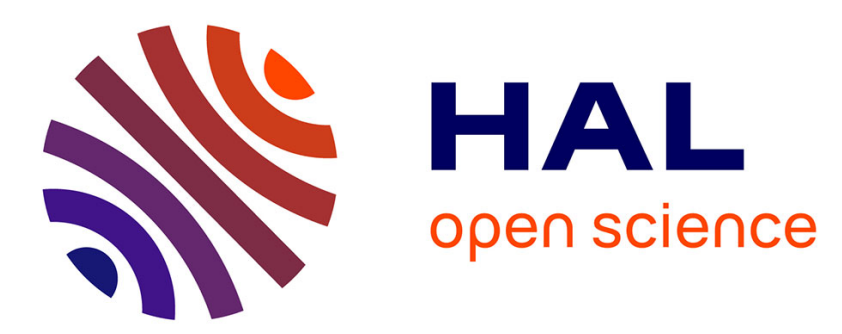

\title{
L'échelle de besoin de récupération : adaptation et validation françaises
}

Christine Bocerean, Emilie Dupret, Christine Bocéréan, Martine Feltrin, Élise Chemolle, Julie Lebon

\section{- To cite this version:}

Christine Bocerean, Emilie Dupret, Christine Bocéréan, Martine Feltrin, Élise Chemolle, et al.. L'échelle de besoin de récupération : adaptation et validation françaises. Santé Publique, 2018, 30 (4), pp.445. 10.3917/spub.185.0445 . hal-02480466

\section{HAL Id: hal-02480466 https://hal.science/hal-02480466}

Submitted on 20 Feb 2020

HAL is a multi-disciplinary open access archive for the deposit and dissemination of scientific research documents, whether they are published or not. The documents may come from teaching and research institutions in France or abroad, or from public or private research centers.
L'archive ouverte pluridisciplinaire HAL, est destinée au dépôt et à la diffusion de documents scientifiques de niveau recherche, publiés ou non, émanant des établissements d'enseignement et de recherche français ou étrangers, des laboratoires publics ou privés. 


\title{
L'ÉCHELLE DE BESOIN DE RÉCUPÉRATION : ADAPTATION ET VALIDATION FRANÇAISES
}

Émilie Dupret, Christine Bocéréan, Martine Feltrin, Élise Chemolle, Julie Lebon

\author{
S.F.S.P. | « Santé Publique »
}

2018/4 Vol. 30 | pages 445 à 454

ISSN 0995-3914

Article disponible en ligne à l'adresse :

https://www.cairn.info/revue-sante-publique-2018-4-page-445.htm

\section{Pour citer cet article :}

Émilie Dupret et al., « L'échelle de besoin de récupération : adaptation et validation françaises », Santé Publique 2018/4 (Vol. 30), p. 445-454.

DOI $10.3917 /$ spub.185.0445

Distribution électronique Cairn.info pour S.F.S.P..

(C) S.F.S.P.. Tous droits réservés pour tous pays.

La reproduction ou représentation de cet article, notamment par photocopie, n'est autorisée que dans les limites des conditions générales d'utilisation du site ou, le cas échéant, des conditions générales de la licence souscrite par votre établissement. Toute autre reproduction ou représentation, en tout ou partie, sous quelque forme et de quelque manière que ce soit, est interdite sauf accord préalable et écrit de l'éditeur, en dehors des cas prévus par la législation en vigueur en France. Il est précisé que son stockage dans une base de données est également interdit. 


\title{
L'échelle de besoin de récupération : adaptation et validation françaises
}

\section{The Need For Recovery Scale: French adaptation and validation}

\author{
Émilie Dupret ${ }^{1}$, Christine Bocéréan ${ }^{2}$, Martine Feltrin ${ }^{1}$, Élise Chemolle ${ }^{1}$, Julie Lebon ${ }^{1}$
}

\section{$\sqsubset \rightarrow$ Résumé}

Objectifs : L'étude présente la validation française de l'échelle de besoin de récupération après le travail (Need For Recovery) ainsi que les scores moyens selon le sexe, l'âge, l'activité professionnelle et le secteur d'activité.

Méthode : Un échantillon représentatif des salariés français a complété un questionnaire composé de l'échelle de besoin de récupération, de l'échelle de stress perçu (Perceived Stress Scale), du questionnaire de symptômes anxieux et dépressifs (Hospital Anxiety and Depression Scale) et d'un questionnaire de burn out (Maslach Burnout Inventory). Les analyses de consistance interne nous ont amenés à retirer un item de l'échelle originelle ; la validité concourante ainsi que la qualité discriminante ont été évaluées avec les analyses adéquates.

Résultats : La version française de l'échelle de besoin de récupération possède de très bonnes qualités psychométriques. La consistance interne (évaluée par l'Alpha de Cronbach) est de 0,86 . Cette échelle est corrélée principalement à la dimension «épuisement émotionnel » du questionnaire de burn out $(r=0,68)$. Les analyses de différence de moyennes ont mis en évidence des groupes de salariés français ayant des niveaux de besoin de récupération élevés : les hommes de moins de 25 ans, les femmes de plus de 55 ans et les répondants exerçant l'activité professionnelle de « Instituteurs et assimilés ».

Conclusions: Étant donné sa valeur prédictive sur la santé physique et psychologique des salariés, nous préconisons une utilisation individuelle ou collective de l'échelle besoin de récupération afin de mettre en place des actions de prévention sur les lieux de travail.

Mots-clés: Besoin de récupération; Études de validation; Risques psychosociaux; Stress psychologique; Prévention primaire.

\section{$\lceil\rightarrow$ Abstract}

Objectives: The study presents the French validation of the scale of Need for Recovery after work (Need for Recovery) as well as the average scores according to sex, age, occupation and branch of industry.

Method: A representative sample of the French employees completed a questionnaire made up of the Need for Recovery Scale, Perceived Stress Scale, Hospital Anxiety and Depression Scale and Maslach Burnout Inventory - General Survey. Analyses of internal consistency led us to withdraw an item from original scale; validity as well as discriminating quality were evaluated with the adequate analyses.

Results: The French version of the Need for Recovery Scale has very good psychometric qualities. Internal consistency (evaluated by Alpha of Cronbach) is 0.86. This scale is correlated mainly with the "emotional exhaustion" dimension of the Maslach Burnout Inventory $(r=0.68)$. The analysis highlighted groups of French employees having levels of high need for recovery: men of less than 25 years, women of more than 55 years and people practicing the profession of 'Teachers and equivalents.'

Conclusions: Given its predictive value on the physical and psychological health of employees, we recommend an individual or collective use of the Need for Recovery Scale in order to set up preventive actions in the workplaces.

Keywords: Need for Recovery; Validation studies; Psychosocial risks; Psychological stress; Primary prevention.

\footnotetext{
${ }^{1}$ PREVENTIS - Centre d'Intervention pour la Santé au Travail - 12-14, rue Jean-Nicot - 75007 Paris - France.

${ }^{2}$ Université de Lorraine - Campus Lettres et Sciences Humaines - Département de Psychologie - 23, boulevard Albert $1{ }^{\text {er }}-$ BP 60446 - 54001 Nancy cedex - France.
} 


\section{Introduction}

La notion de prévention des risques psychosociaux est désormais devenue de plus en plus familière à l'ensemble des acteurs de la santé au travail, aux instances représentatives internes aux entreprises, aux salariés et à leurs employeurs. En France, cette prise en compte a été rapide ${ }^{1}$, peut-être parce qu'elle a été relativement tardive par rapport à d'autres pays européens, essentiellement nordiques. L'obligation pour les employeurs de prendre en compte le bien-être au travail des salariés marque une avancée importante dans la protection de la santé au travail. Néanmoins, la maîtrise de ce que recouvre la notion de risques psychosociaux ne semble pas tout à fait réalisée, peut-être en raison du manque de recul français. Si les facteurs de risque ont bien été définis dans le rapport dit «Gollac» [1], les conséquences possibles de l'exposition à ces facteurs de risque - ce que nous pouvons appeler les «troubles » psychosociaux - le sont beaucoup moins. On assiste alors à un foisonnement de concepts, fortement médiatisés via des résultats d'enquêtes dont on ne maîtrise pas nécessairement le caractère scientifique, ni surtout la représentativité de la population étudiée : burn out, épuisement professionnel, sur-stress, workaholisme, dépression, fatigue chronique, voire bore-out... Ces informations sont parfois contradictoires voire, comparées à celles émanant d'autres pays européens, sans décrire une quelconque méthodologie d'enquête.

Il convient donc de posséder des outils fiables, en l'occurrence de questionnaires validés scientifiquement et largement utilisés dans d'autres pays afin, d'une part, de définir précisément les concepts qui sont mesurés et, d'autre part, de présenter des résultats représentatifs de la population française active. Ainsi, nous avons contribué à la validation de l'échelle de stress perçu de Cohen en quatre items (Perceived Stress Scale - PSS) [2] et de l'Hospital Anxiety and Depression Scale (HADS) auprès d'une population représentative de salariés français [3]. Nous avons également introduit et validé en France le Copenhagen Psychosocial Questionnaire (COPSOQ) [4, 5].

Dans l'idée d'adopter une démarche positive, c'est-à-dire qui vise à relever les potentialités des salariés plutôt que leur fragilité, nous avons recherché un questionnaire utilisé

\footnotetext{
${ }^{1}$ Le premier accord intersyndical national entre salariés et employeurs portant sur le «stress au travail » a été conclu il n'y a qu'une dizaine d'années; il s'agit de l'Accord national interprofessionnel sur le stress au travail du 2 juillet 2008.
}

depuis longtemps dans des pays européens et qui s'attache à mesurer non plus les facteurs de risque, mais les facteurs de protection. Cet article présente ainsi la validation française de l'échelle Need For Recovery (NFR), introduite en 1994 aux Pays-Bas par Van Veldhoven et Meijman [6].

Nous exposons dans un premier temps les concepts sousjacents à la notion de besoin de récupération, sa mesure et discutons de ses liens avec des variables liées à la santé psychologique des salariés.

\section{De la fatigue au travail au besoin de récupération}

Bien que la notion de fatigue liée au travail ait intéressé les chercheurs depuis la fin du XIX ${ }^{e}$ siècle, le premier modèle et la première théorisation ont été effectués par Meijman [7, 8] en 1989. L'auteur a mis en évidence les processus biologiques, motivationnels, cognitifs et émotionnels impliqués dans l'expérience de fatigue et a surtout déplacé le concept de fatigue pendant le travail à celui du ressenti de fatigue à la fin ou juste après une journée de travail. En effet, le ressenti de fatigue après une journée de travail affecte non seulement la capacité à absorber, le jour suivant, les contraintes du travail, mais également la qualité de la vie privée.

Dans le modèle «Effort/Récupération » présenté par Van Veldhoven [9, p. 2] et basé sur les travaux de Meijman [7], Van Veldhoven [10] et de Meijman et Mulder [11], les contraintes classiques d'une journée de travail induisent des coûts physiques, cognitifs et psychologiques, mais ces contraintes s'arrêtent lorsque la journée de travail est terminée. Les conséquences de ces coûts sont, d'après Meijman et Schaufeli [12], ce que l'on pourrait appeler la fatigue à court terme. Le modèle est multifactoriel, car ces contraintes sont largement influencées par des caractéristiques du travail (charge, rythme, mais également la possibilité ou non d'une forme de liberté et d'influence dans son travail - faire des pauses, prendre des vacances quand le salarié le désire, maîtriser ses horaires de travail) et évidemment des caractéristiques situationnelles et individuelles.

La notion de fatigue après le travail implique la manifestation de plusieurs symptômes à la fin de la journée de travail et pas seulement la seule fatigue physique. Glass et Singer [13], Mohr [14] ou encore Jansen, Kant et Van Den Brandt [15] l'ont liée au concept «d'irritabilité après le travail ». Meijman [8] parle de pression psychologique, d'irritabilité, de retrait social, de manque d'énergie et de réduction de la volonté pour fournir un nouvel effort, des niveaux de performance réduits et d'un état de fatigue 
physique. Si les manifestations dues au stress peuvent être décrites, entre autres, par ce même type de symptômes, conceptuellement il existe au moins une distinction importante entre le stress et la fatigue après le travail : dans le cas des manifestations décrites par les auteurs cités précédemment, il s'agit de comportements classiques observés après une journée de travail ; dans le cas d'un fonctionnement typique de la personne, ces troubles disparaissent par le fait même de la récupération. Dans le cas du stress, ces comportements, ces ressentis ne peuvent disparaître en une nuit, sont installés et entrent dans la catégorie du trouble psychosocial.

Idéalement, le processus de récupération de la fatigue induite par une journée de travail peut s'opérer pendant la journée de travail, à la fin de la journée et dans la nuit. Dans ce cas, le salarié débutera une nouvelle journée en ne présentant plus de symptômes liés aux contraintes de la journée précédente. Par contre, si les conditions de récupération n'ont pas pu être satisfaisantes, c'est-à-dire si la récupération en tant que facteur de protection a échoué, Kompier [16] suppose un «effet cumulatif » qui rend le besoin de récupération de plus en plus présent et qui exige, chaque jour, un effort supplémentaire de la part du salarié. C'est le début d'un cercle vicieux.

\section{Liens entre le besoin de récupération et d'autres variables relatives aux risques psychosociaux}

Jansen, Kant et Van der Brandt [23] ont analysé les réponses de plus de 12000 salariés néerlandais (issus de 45 entreprises ou institutions) au questionnaire VBBA et ont observé des corrélations significatives entre les scores à l'échelle NFR et ceux estimant les contraintes psychologiques du travail, la latitude décisionnelle, la pénibilité du travail, ainsi que les scores à l'échelle de fatigue Checklist Individual Strength (CIS) et au General Health Questionnaire (GHQ-12). Sluiter, De Croon, Meijman et Frings-Dresen [24] ont identifié des liens élevés entre les contraintes du travail et les scores de besoin de récupération pour différents métiers, résultats provenant de six études distinctes menées entre 1996 et 2002. Kiss et De Meester [25] ont étudié plus particulièrement les résultats selon l'âge : si la charge cognitive et émotionnelle au travail est liée à un risque plus élevé de besoin de récupération pour tous, la relation avec les collègues semble jouer un rôle plus important (sur le score de besoin de récupération) en ce qui concerne les salariés les plus jeunes.

Dans leur étude sur une population représentative des salariés allemands, Sonnentag et Zijlstra [26] ont montré que des contraintes quantitatives élevées au travail (charge de travail, travail dans l'urgence, investissement, heures supplémentaires), qu'une latitude décisionnelle faible (responsabilités, possibilités de contrôle) ainsi que des activités extra-professionnelles insatisfaisantes prédisaient un score élevé de besoin de récupération et une mauvaise évaluation du bien-être.

Les liens entre le besoin de récupération, le conflit vie professionnelle/vie privée ainsi que la performance au travail ont été étudiés de façon longitudinale par Demerouti, Taris et Bakker [27]. Le besoin de récupération et le conflit vie professionnelle/vie privée interagissent l'un sur l'autre, avec un impact sur la performance au travail.

$\mathrm{Au}$ niveau physiologique, l'étude de Sluiter, FringsDresen, Van Der Beek et Meijman [28] a établi un lien entre le besoin de récupération, l'auto évaluation de la santé et les taux d'adrénaline et de cortisol : plus le score de besoin de récupération est élevé, plus les taux d'adrénaline et de cortisol le sont et plus les problèmes de santé sont importants, résultats retrouvés dans l'étude de Qi, Liu, Zhang, Ji, Sluiter, Zhou, et al. [29].

Enfin, des auteurs se sont intéressés à la valeur prédictive du score de NFR. De Croon, Sluiter et Frings-Dresen [30] ont montré que le niveau de besoin de récupération prédit les arrêts maladie supérieurs à 14 jours prescrits deux années plus tard; de la même façon, le score de besoin de récupération prédit les troubles de santé (en termes de troubles psychosomatiques, d'épuisement émotionnel ou de problèmes de sommeil) [24], le taux de turn-over [31], les maladies cardiovasculaires [32] et des troubles musculo squelettiques [33].

\section{La mesure du besoin de récupération}

Les premières études pour mesurer le besoin de récupération ont été menées par Meijman, Cavalini, Van Dormolen, Gloerich, Hellinga et Van Noord [19] ainsi que par Meijman [7] aux Pays-Bas. Le questionnaire utilisé était couplé à une évaluation des contraintes quantitatives du travail. L'analyse des qualités psychométriques de ce premier questionnaire a conduit les auteurs à proposer l'échelle Need For Recovery after work, échelle réduite à 11 items [6]. Cette échelle est un sub-test du questionnaire «Vragenlijst Beleving en Beoodeking van de Arbeid» (VBBA), utilisé dès 1994 par les services de médecine du travail néerlandais, questionnaire qui explore, avec 27 échelles, ce que nous appelons maintenant les risques psychosociaux en interrogeant les facteurs de risque et leurs conséquences sur la santé physique et mentale des individus. 
Les modalités de réponse aux 11 items de l'échelle NFR sont dichotomiques (oui/non). L'échelle NFR évalue les difficultés à récupérer son énergie après une journée de travail et interroge aussi bien les symptômes relatifs à la fatigue physique et mentale que le temps nécessaire pour revenir à un niveau de fonctionnement normal. Un score sur 11 points est obtenu en additionnant les réponses (après recodage des items inversés) ; il est transformé en un score sur 100. Broersen, Fortuin, Dijkstra, Van Veldhoven et Prins [20] estiment que les salariés obtenant un score supérieur à 55 présentent un risque accru d'absence pour maladie due à des problèmes de santé physique ou psychologique. Les qualités psychométriques de l'échelle NFR sont présentées en détail par Van Veldhoven [9].

Des traductions en anglais de cette échelle ont été éditées par Sluiter, Van Der Beek et Frings-Dresen [21] et par Van Veldhoven et Broersen [22] ; traduit et validé dans plusieurs pays européens (Allemagne, Belgique, Norvège, Italie), l'échelle NFR a fait l'objet de recherches qui ont permis de tester les liens entre un score élevé de besoin de récupération et de nombreux facteurs de risque ainsi que la valeur prédictive des scores élevés.

Le questionnaire VBBA a été traduit en français pour les salariés francophones belges. La version française utilisée en Belgique n'est pas publiée et les études se centrent sur la comparaison entre les versions française et néerlandaise.

\section{Méthode}

\section{Élaboration de la version française de l'échelle NFR}

Les 11 items de l'échelle NFR ont été soumis au processus de «translation/ back translation » à partir de la version anglaise publiée dans Van Veldhoven [9]. La formulation des items en français a été testée auprès de 30 personnes volontaires afin de vérifier la bonne compréhension des items : deux psychologues leur ont demandé de compléter les questions et de les interpréter. La structure de certaines phrases a ainsi été revue en tenant compte des remarques des personnes interrogées. La version modifiée a, à nouveau, fait l'objet de la même démarche qualitative auprès de 20 personnes : elle a été comprise et interprétée de la même façon par toutes les personnes interrogées. La version française définitive de l'échelle NFR a été nommée échelle de besoin de récupération (BDR).

\section{Contenu du dossier de questionnaires}

Le dossier de questionnaires complet proposé aux participants comportait l'échelle BDR, le Maslach Burnout Inventory - General Survey (MBI-GS) [34] en cours de validation française, la PSS en dix items [2] et l'HADS [3].

L'échelle BRD est constituée de 11 items dont les modalités de réponse sont dichotomiques (oui ou non). La consigne était la suivante: "Les affirmations suivantes concernent le besoin de récupération. Indiquez, par oui ou par non, ce que vous ressentez le plus souvent ». Les participants ont également complété les variables signalétiques décrites ci-dessous.

\section{Population}

Un échantillon de 1449 salariés a été interrogé par l'intermédiaire de notre partenaire Opinionway (Institut d'études et de sondage). L'ensemble des répondants est représentatif (par la méthode des quotas et par la pondération de chaque répondant) de la population salariée française au niveau du sexe, de l'âge, du secteur d'activité, de l'activité professionnelle, de la fonction d'encadrement et, le cas échéant, du nombre de salariés encadrés. Le recueil des données a eu lieu en avril 2016.

\section{Étapes d'analyse}

Nous avons mené une série d'analyses avec le logiciel SPSS 22.0 afin d'évaluer les qualités psychométriques de l'échelle BDR. Après avoir vérifié que la distribution des données suivait la loi normale, nous avons dans un premier temps calculé la consistance interne de l'échelle avec le coefficient Alpha de Cronbach. À des fins de vérification, nous avons également soumis les données à une Analyse factorielle exploratoire à Composantes Principales (ACP) avec comme critère pour déterminer le nombre de facteur celui des valeurs propres supérieures à 1 ; la rotation choisie est orthogonale (varimax).

Nous avons ensuite vérifié la validité concourante de la mesure du besoin de récupération en calculant des corrélations avec les scores aux MBI-GS, à la PSS et à l'HADS.

Enfin, nous avons mené des analyses de différences de moyennes afin d'observer si le score de besoin de récupération différenciait des groupes de personnes selon les critères interrogés et afin de présenter les scores moyens de BDR par sous-groupes en tant que "normes» françaises. 


\section{Résultats}

\section{Consistance interne de l'échelle BDR et ACP}

Lors de l'analyse de consistance interne de l'échelle BDR à 11 items, il s'est avéré que l'item 10 («Après le repas du soir, je me sens généralement encore assez en forme ») faisait chuter le coefficient Alpha de Cronbach ( $c f$. Tableau I). Nous avons donc décidé de l'exclure de l'échelle. Le coefficient Alpha de Cronbach, calculé sur la version à dix items, est de 0,86. Ce coefficient, calculé pour chacun des sousgroupes définis par les variables signalétiques (à l'exception de l'activité professionnelle dont l'effectif de certaines modalités est relativement faible), varie de 77 (pour répondants âgés de moins de 25 ans) à .90 (pour les répondants âgés de 55 à 59 ans).

Tableau I : Coefficients Alpha de Cronbach $(\alpha)$ en cas de suppression de l'élément pour l'échelle en 11 items et en 10 items

\begin{tabular}{lcc}
\hline \multicolumn{1}{c}{ Items } & $\begin{array}{c}\alpha \\
11 \text { items }\end{array}$ & $\begin{array}{c}\alpha \\
10 \text { items }\end{array}$ \\
\hline $\begin{array}{l}\text { À la fin d'une journée de travail, je me sens } \\
\text { vraiment épuisé(e). }\end{array}$ & 0,778 & 0,841 \\
\hline $\begin{array}{l}\text { J'ai peu d'intérêt pour les autres lorsque je } \\
\text { viens de rentrer à la maison, après le travail. }\end{array}$ & 0,792 & 0,852 \\
\hline $\begin{array}{l}\text { À cause de mon travail, je me sens plutôt } \\
\text { exténué(e) à la fin de la journée. }\end{array}$ & 0,775 & 0,837 \\
\hline $\begin{array}{l}\text { En général, je ne me détends qu'à partir } \\
\text { du deuxième jour de repos. }\end{array}$ & 0,789 & 0,851 \\
\hline $\begin{array}{l}\text { Souvent, après une journée de travail, } \\
\text { la fatigue m'empêche de m'investir dans } \\
\text { d'autres activités. }\end{array}$ & 0,776 & 0,838 \\
\hline $\begin{array}{l}\text { J'ai des difficultés à me concentrer pendant } \\
\text { mes heures de loisirs après le travail. }\end{array}$ & 0,783 & 0,844 \\
\hline $\begin{array}{l}\text { Généralement, j'ai besoin de plus d'une } \\
\text { heure pour récupérer complètement après }\end{array}$ & 0,777 & 0,840 \\
\hline $\begin{array}{l}\text { le travail. } \\
\text { Quand je rentre du travail, j'ai besoin qu'on } \\
\text { me laisse tranquille pendant un certain } \\
\text { temps. }\end{array}$ & 0,785 & 0,847 \\
\hline $\begin{array}{l}\text { Il arrive que dans la dernière partie de } \\
\text { la journée de travail, la fatigue m'empêche } \\
\text { de faire mon travail aussi bien. }\end{array}$ & 0,794 & 0,855 \\
\hline $\begin{array}{l}\text { Après le repas du soir, je me sens } \\
\text { généralement encore assez en forme. }\end{array}$ & & \\
\hline $\begin{array}{l}\text { J'ai des difficultés à me détendre à la fin } \\
\text { d'une journée de travail. }\end{array}$ & 0,780 & 0,842 \\
\hline & & \\
\hline & & \\
\hline & & \\
\hline
\end{tabular}

L'ACP aboutit à un seul facteur, expliquant 44,3\% de la variance.

\section{Calcul des scores et description de la distribution obtenue}

L'échelle BDR est constituée de dix items codés 0 ou 1. Un score est calculé pour chaque participant, multiplié par 10 , afin d'obtenir un score variant de 0 à 100 . Plus le besoin de récupération est élevé, plus le score l'est également. La distribution obtenue est une distribution normale (Test de Kolmogorov-Smirnov avec correction de signification de Lilliefors, $p=0,000)$. La moyenne est de 47,95, l'écart type de 32,55 .

\section{Validité concourante}

Pour chaque participant, nous avons également calculé un score aux trois échelles du questionnaire MBI-GS, un score de stress (PSS), un score d'anxiété (HADS) et un score de dépression (HADS). Le score de BDR est corrélé positivement $(\mathrm{p}<0,01)$ avec le score d'épuisement émotionnel du MBI-GS ( $r=0,68)$, avec le score de cynisme du MBI-GS $(0,39)$, avec le score de stress $(r=0,56)$, avec le score de symptômes dépressifs $(r=0,53)$ et avec le score de symptômes anxieux $(\mathrm{r}=0,50)$. Il est corrélé négativement $(\mathrm{p}<0,01)$ avec le score d'efficacité professionnelle du MBI-GS $(r=-0,18)$.

\section{Analyse discriminante}

Nous avons effectué des analyses de variance selon les variables signalétiques interrogées. Nous n'avons observé aucune différence significative en ce qui concerne la fonction ou non d'encadrement ainsi que le nombre de personnes encadrées. Par contre, les scores moyens de besoin de récupération se différencient selon le sexe, l'âge, l'activité professionnelle ainsi que le secteur d'activité.

\section{Différence selon le sexe}

Les femmes $(M=51$; ET = 33) présentent un score moyen de besoin de récupération supérieur à celui des hommes $(\mathrm{M}=45 ; \mathrm{ET}=31 ; \mathrm{F}(1,1444)=14,9 ; \mathrm{p}<0,01)$.

\section{Différence selon l'âge}

Nous présentons les différences selon les groupes d'âge en fonction du sexe car le test des effets inter-sujets montre 
une interaction entre le sexe et l'âge ( $\mathrm{p}=0,002 ; c f$. Figure 1$)$. Dans les deux cas (homme et femme), les groupes se différencient selon l'âge (resp. $F(8,736)=3,29$, p < 0,01 et $F(8,693)=2,32, p<0,04)$.

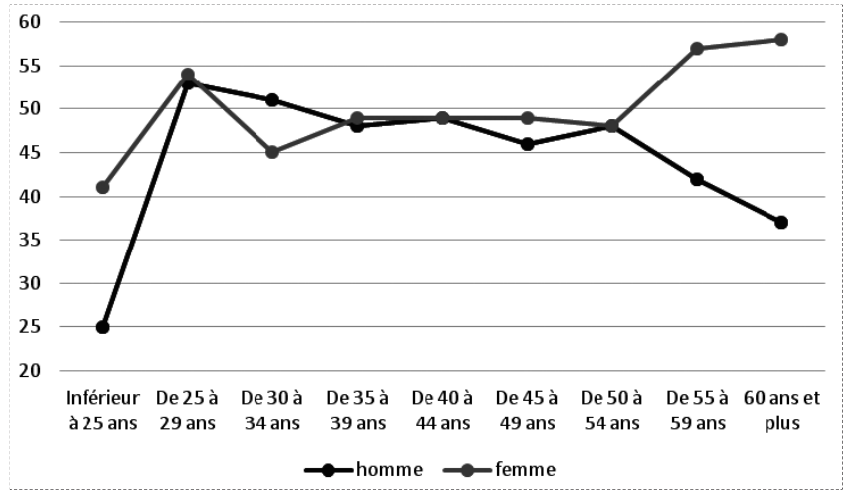

Figure 1 : Moyennes des scores BDR par groupe d'âge selon le sexe

\section{Secteur d'activité}

Les salariés se différencient également selon le secteur d'activité de leur entreprise ou institution $(F(9,1422)=$ 3,$49 ; \mathrm{p}<0,01$ ) ; le secteur « Agriculture » ne figure pas dans cette analyse $(n=14)$. Les salariés du BTP se distinguent nettement et favorablement des salariés des autres secteurs d'activité avec le score moyen de besoin de récupération le plus bas ( $c f$. Figure 2).

Si on différencie les salariés travaillant dans le secteur public et ceux dans le secteur privé, on observe aucune spécificité selon le secteur.

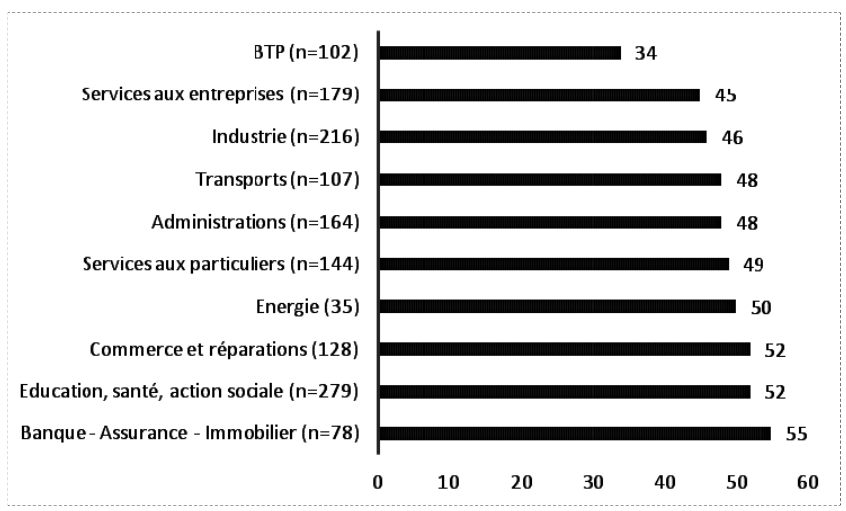

Figure 2 : Moyennes des scores BDR selon le secteur d'activité

\section{Discussion}

\section{Qualités psychométriques de l'échelle BDR}

Comme nous l'avons écrit précédemment, l'échelle besoin de récupération, dans sa version française, est composée de 10 items et non pas de 11 . L'item que nous avons exclu concerne la fatigue ressentie après le repas du soir. Il en a été de même dans la validation de la version italienne [35] : les auteurs expliquent que le temps «après le repas du soir » est plus long dans les pays nordiques qu'en Italie. Nous pouvons reprendre cette explication à notre compte et la préciser : en effet, le dîner, de façon générale, a plutôt lieu vers 18 heures dans les pays nordiques et après 20 heures dans les pays méditerranéens, y compris en France. Le type d'activité pouvant se dérouler « après le repas du soir » et le degré de fatigue ne sont pas équivalents selon les pays [9]. Pace, Lo Cascio, Civilleri, Guzzo, Foddai et Van Veldhoven [35] suggèrent de réfléchir à une autre formulation pour cet item. De notre point de vue, étant donné que la version en 10 items présente des qualités psychométriques satisfaisantes et que l'échelle est unidimensionnelle, nous estimons qu'il n'est pas nécessaire d'élaborer un item supplémentaire.

Les coefficients de consistance interne, dans cette étude, pour l'ensemble des répondants ou pour les sous-groupes sont similaires à ceux publiés dans la littérature qui varient de 0,78 [15] à 0,88 [22] - en ce qui concerne l'ensemble des répondants - et de 0,81 à 0,92 [22] pour des sous-groupes. De même, le pourcentage de variance expliquée dans l'ACP que nous avons menée est semblable aux résultats obtenus dans d'autres études [e.g. 35].

La validité concourante de l'échelle NFR a été testée, dans la littérature, avec principalement deux questionnaires. Le premier est l'échelle de fatigue subjective, subtest de la Checklist Individual Resilience [36]. La corrélation entre les deux échelles est de 0,66 dans une étude portant sur des salariés du secteur de la construction [37] et de 0,71 dans une autre étude concernant le secteur des transports [38]. Ce sont des corrélations attendues étant donné que le concept de besoin de récupération inclut la notion de fatigue.

Par contre, les auteurs se sont étonnés des corrélations élevées entre l'échelle NFR et deux des dimensions de la Utrecht Burnout Scale (UBOS) [39] ; ainsi, le score de besoin de récupération et le score à la dimension d'épuisement émotionnel du questionnaire UBO sont corrélés à 0,75 dans une population en contact avec le public [22] et à 0,80 dans 
une population de salariés tout venant [39]. De plus, Sonnentag et Fritz [40] ont bien retrouvé cette corrélation entre le score de NFR et la même dimension d'épuisement émotionnel $(0,82)$ mais également un lien entre le score de NFR et la dimension de détachement psychologique de l'UBOS $(-0,52)$. Les corrélations que nous avons obtenues entre le score de BDR et l'échelle d'épuisement émotionnel du MBI-GS $(0,68)$ ainsi que celle que nous nommons « cynisme » - et qui semble correspondre à la dimension de détachement émotionnel du questionnaire UBO - (0.39) sont tout à fait cohérentes avec les résultats publiés dans la littérature. Néanmoins, il ne faut pas oublier que le concept de burn out comporte trois dimensions et que, même si nous avons obtenu une corrélation significative et négative (étant donné la taille de notre échantillon) avec la troisième dimension (efficacité professionnelle), nous ne pouvons absolument pas affirmer que ce que mesure l'échelle de besoin de récupération recouvre les trois dimensions du burn out. Néanmoins, dans la théorie la plus consensuelle du développement du burn out [41], la première étape est un état d'épuisement émotionnel. Boudoukha [42, p. 25] illustre cet état en expliquant que, pour une personne souffrant d'épuisement émotionnel, ce «manque d'énergie est d'autant plus fort que l'individu pense qu'il n'a aucun moyen à sa disposition pour "recharger ses batteries". La seule pensée d'avoir à affronter une nouvelle journée au travail dans ces conditions lui est insupportable». Cette description correspond fortement à ce que peut ressentir une personne dont le processus de récupération a échoué. Ainsi, un score élevé à l'échelle besoin de récupération pourrait être un indicateur précurseur d'un état d'épuisement émotionnel, si ce n'est un premier signe de risque de burn out.

Les auteurs présentent également des résultats concernant les corrélations entre le score de NFR et des questionnaires de santé (e.g. [15] $\mathrm{r}=0,48$ avec le GHQ-12), des questions sur des troubles psychosomatiques ([40] $\mathrm{r}=$ $0,52)$ et des symptômes dépressifs ([40] $r=0,55)$. Nous avons également retrouvé de tels liens : dans cette étude, le score de BDR est corrélé à 0,56 avec le score de stress, à 0,53 avec le score de symptômes dépressifs et à 0,50 avec le score de symptômes anxieux.

Toutes ces corrélations semblent confirmer, d'une part la complexité de la notion de besoin de récupération après le travail et, d'autre part son importance, étant donné sa comorbidité avec des troubles psychologiques sérieux. Pour illustrer ces propos, le tableau II présente les scores moyens de BDR selon la répartition en seuil des scores de symptômes anxieux et dépressifs [3]. Les répondants présentant un score élevé de symptômes anxieux ont un
Tableau II : Moyenne et écart type à l'échelle de besoin de récupération selon les scores à l'HADS

\begin{tabular}{lccc}
\hline \multicolumn{1}{c}{ Scores à I'HADS } & \multicolumn{3}{c}{ Scores de Besoin De Récupération } \\
\hline Symptômes anxieux & Moyenne & Écart type & \% de salariés \\
\hline$<8$ & 36,90 & 29,70 & 57,80 \\
\hline Entre 8 et 10 & 59,30 & 29,75 & 20,40 \\
\hline$>10$ & 66,84 & 29,19 & 21,80 \\
\hline \multicolumn{1}{c}{$\begin{array}{c}\text { Symptômes } \\
\text { dépressifs }\end{array}$} & & & \\
\hline$<8$ & 37,53 & 29,55 & 62,20 \\
\hline Entre 8 et 10 & 58,55 & 30,18 & 22,40 \\
\hline$>10$ & 73,29 & 27,30 & 15,40 \\
\hline
\end{tabular}

score moyen de près de 67 à l'échelle BDR ; ceux présentant un score élevé de symptômes dépressifs ont un score moyen de plus de 73 à l'échelle BDR, ce qui représente, dans les deux cas, des niveaux très élevés de besoin de récupération.

\section{Scores moyens de la population salariée française}

Le score moyen de BDR, calculé sur un échantillon représentatif des salariés français est de 47,95 (écart type de $32,55)$. C'est la valeur moyenne la plus élevée que l'on rencontre dans la littérature : toutes les études néerlandaises, allemandes ou belges présentent des scores moyens inférieurs. Seule l'étude de validation italienne de 2013 décrit un score moyen équivalent [35].

La différence entre les femmes et les hommes, en défaveur des femmes, a été retrouvée dans notre étude conformément à la littérature [15, 35, 43]. Comme dans l'étude italienne, les femmes salariées françaises ont six points de plus que les hommes. Ces résultats soulignent probablement un phénomène d'inégalité souvent pointée par les femmes salariées qui accomplissent une double journée de travail : un travail salarié la journée et un travail domestique la soirée. Ils sont cohérents avec ceux obtenus avec d'autres questionnaires de santé psychologiques [2,3].

Les scores moyens selon l'âge sont plus complexes à comparer avec les résultats de la littérature car les groupes d'âge ne sont pas toujours identiques. Nous allons d'abord nous centrer sur les similitudes et différences entre les hommes et les femmes de notre échantillon avant d'effectuer des comparaisons avec les résultats des autres recherches. 
Dans notre étude, les scores des hommes et des femmes âgés de 25 à 54 ans ne diffèrent pas selon le sexe. Les deux grandes différences concernent les plus jeunes et les plus âgés. Ainsi, même si le score moyen des femmes âgées de moins de 25 ans est plus élevé que celui des hommes du même âge, ces scores sont les plus bas pour chacun des groupes, comme on peut l'observer sur la figure 1 . Ce résultat est similaire à celui décrit par Mohren, Jansen et Kant [43] mais différent de celui obtenu par Kiss, De Meester et Braeckman [44] qui consistait en un score élevé (pas le plus élevé, certes) des répondants de 25 ans et moins. Ces auteurs expliquaient cet important besoin de récupération par le manque d'expérience des jeunes salariés. Dans notre étude, ce sont les salariés âgés de 25 à 29 ans qui présentent des scores élevés et l'augmentation entre les moins de 25 ans et les 25 à 29 ans est particulièrement forte chez les hommes. En ce qui concerne les salariés les plus âgés, la courbe des hommes correspond à ce qui est décrit dans la littérature : on observe une baisse des scores après 55 ans $[43,44]$. Plusieurs interprétations sont données, se rapportant globalement au concept de « travailleur sain » : les salariés les plus âgés ont su s'adapter à leurs conditions de travail en apprenant à se ménager (dans l'étude de Mohren, Jansen et Kant [43] ce sont ces salariés qui effectuaient le moins d'heures supplémentaires) et en ayant mis en œuvre des stratégies de compensation pour minimiser le besoin de récupération grâce à leur expérience acquise et à l'expertise de leur métier. Par contre, la courbe des femmes est très différente de celle des hommes : on observe un besoin de récupération accru à partir de 55 ans ; le niveau atteint à 60 ans et plus est le plus élevé de tous les groupes analysés. Ces résultats ne sont pas décrits dans la littérature, peu d'auteurs ayant analysé séparément les hommes et les femmes.
En conclusion de cette double analyse, nous estimons que les niveaux de besoin de récupération des hommes âgés de moins de 25 ans et des femmes âgées de plus de 55 ans sont préoccupants.

Les différences selon l'activité professionnelle sont également bien marquées ( $c f$. figure 3 ), avec la catégorie «Instituteurs et assimilés » présentant un score de besoin de récupération très élevé (les scores des hommes et des femmes ne présentant pas de différence). Nous n'avons pas de données comparatives; les auteurs, classiquement, comparent les niveaux de diplôme. Dans l'étude de validation italienne, les auteurs [35] observent un score moyen de besoin de récupération plus élevé chez les salariés les moins diplômés et un score plus faible chez les salariés diplômés d'université. Nous ne pouvons pas nous baser sur ces résultats, car les activités professionnelles ayant le score moyen le plus bas et le score moyen le plus élevé nécessitent, toutes les deux, un niveau universitaire. Il nous semble donc que le caractère intrinsèque de l'activité exercée est plus déterminant que le niveau de diplôme.

\section{La question du seuil}

De nombreux questionnaires interrogeant sur la santé physique et mentale possèdent une valeur seuil à partir de laquelle le score obtenu reflète de réelles difficultés pour la personne interrogée. Ceci concerne évidemment une passation individuelle. Dans la littérature, on trouve deux façons de déterminer un seuil en ce qui concerne l'échelle NFR. Broersen, Fortuin, Dijkstra, Van Veldhoven et Prins [20] ont estimé quel était le niveau de besoin de récupération qui révélait un état nécessitant une prise en charge médicale et/ou psychologique : il est de 6 sur 11 soit 55 sur 100 .

Figure 3 : Moyenne des scores BDR selon l'activité professionnelle

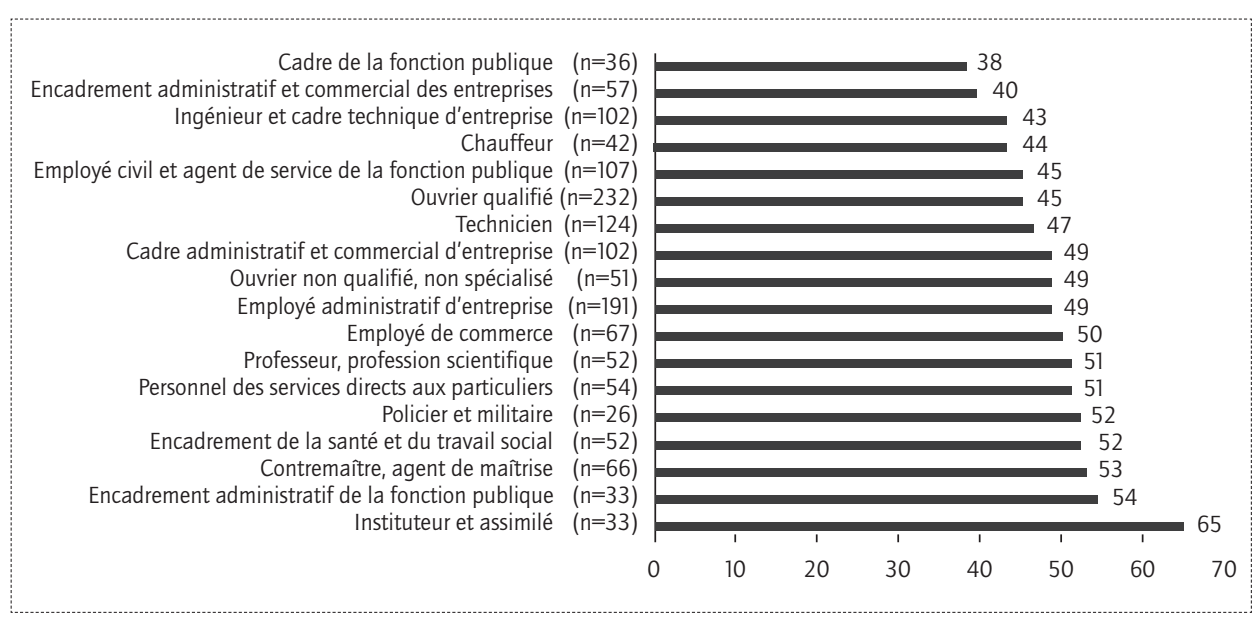


D'autres auteurs, ayant travaillé avec un échantillon représentatif d'une population, ne se basent pas sur un seuil « absolu » mais estiment que les salariés se situant au-delà $\mathrm{du} 3^{\mathrm{e}}$ quartile ont un niveau de récupération trop élevé [e.g. 15]. Étant donné que le score moyen que nous avons obtenu pour la population de salariés français est plus élevé que ceux décrits dans la littérature, le seuil de 55 (qui correspondrait à $43 \%$ des répondants de notre étude) nous semble trop bas. En revanche, si nous nous basons sur la valeur du $3^{\mathrm{e}}$ quartile, c'est-à-dire 80 , nous obtenons un pourcentage de $26,7 \%$ de salariés dont nous pouvons considérer qu'ils ont un niveau élevé de besoin de récupération. Ce seuil de 80 nécessite des études approfondies d'un point de vue clinique.

\section{Conclusion}

\section{Intérêt de cette échelle}

L'échelle besoin de récupération a démontré son intérêt, tant au niveau individuel que collectif, dans différents pays et avec différentes populations. Les nombreuses recherches s'étalant sur plus de 25 ans en témoignent et mettent surtout en exergue le caractère prédictif des scores défavorables. Cette échelle peut ainsi contribuer à la prévention primaire des risques psychosociaux. Elle révèle des symptômes précoces de la fatigue due au travail dont l'accumulation peut aboutir à un épuisement physique et émotionnel ayant évidemment des répercussions sur l'activité professionnelle des salariés.

Dans une optique de prévention, Formanoy, Dusseldorp, Coffeng, Van Mechelen, Boot, Hendriksen etal. [45] ont mené une étude longitudinale pour étudier l'effet d'actions mises en place en entreprise (intervention environnementale ergonomique, groupe de parole sur la motivation, relaxation, formation « travailler et se détendre », etc.). Comme les autres recherches qui ont été effectuées avec cette même méthodologie, les scores de besoin de récupération des salariés ont baissé et cette baisse s'est maintenue au moins pendant un an. Ces études montrent le bien-fondé des actions de prévention qui peuvent être mises en place sur les lieux de travail.

\section{Limites de cette recherche}

Les caractéristiques psychométriques de la version française de l'échelle de besoin de récupération, que nous avons pu analyser, sont très bonnes et conformes aux versions des autres pays utilisant cette échelle. Même si peu de données concernent, à l'étranger, la stabilité (test/retest), elle n'a pas pu être mise en œuvre dans notre étude, étant donné que tous les répondants étaient anonymes.

La littérature sur le concept de besoin de récupération est vaste et de nombreuses variables ont été étudiées conjointement pour essayer de le cerner. Il s'agit maintenant d'effectuer le même type de recherches en France, en tenant compte de la multitude de facteurs interagissant avec le besoin de récupération : caractéristiques du travail, facteurs individuels, interaction entre vie professionnelle et vie privée d'une part, et autres troubles psychosociaux d'autre part.

\section{Aucun conflit d'intérêt déclaré}

\section{Références}

1. Collège d'expertise sur le suivi des risques psychosociaux au travail. Mesurer les facteurs psychosociaux de risque au travail pour les maîtriser. 2011 ; http://www.collegerisquespsychosociaux-travail.fr.

2. Dupret E, Bocéréan C. La mesure du stress en milieu professionnel avec l'échelle de stress perçu (Perceived Stress Scale) : pertinence des versions en dix et quatre items. Psychol Trav Organ. 2013;(19)4:362-84.

3. Bocéréan C, Dupret. A validation study of the Hospital Anxiety and Depression Scale (HADS) in a large sample of French employees. BMC Psychiatry. 2014;14:354.

4. Dupret $E$, Bocéréan $C$, Teherani $M$, Feltrin M. Le COPSOQ : un nouveau questionnaire français d'évaluation des risques psychosociaux. Santé Publique. 2012;24(3):189-207.

5. Dupret E, Bocéréan C, Teherani M, Feltrin M, Petersen J. Psychosocial risks evaluation: French validation of the Copenhagen Psychosocial Questionnaire (COPSOQ). Scand J Public Health. 2012;5:482-90.

6. Van Veldhoven M, Meijman TF. Het meten van psychosociale arbeidsbelasting met een vragenlijst: de vragenlijst beleving en beoordeling van de arbeid (VBBA). Amsterdam : NIA; 1994.

7. Meijman TF (1989). Belasting en herstel: een begrippenkader voor arbeids-psychologisch onderzoek van werkbelasting. In: Meijman TF, editor. Mentale Belasting en Werkstress: een Arbeidspsychologische Benadering. Assen/Maastricht: Van Gorcum. 1989:5-20.

8. Meijman TF. Over vermoeidheid. Amsterdam: Studie-centrum Arbeid en Gezondheid. Ph.D. thesis. 1991.

9. Van Veldhoven M. Need for recovery after work: An overview of construct, measurement and research. In: Houdmont J, Leka S, editors. Occupational health psychology: European perspectives on research, education and practice. Nottingham: Nottingham University Press. 2008:1-25.

10. Van Veldhoven M. Psychosociale arbeidsbelasting en werkstress. Lisse: Swets \& Zeitlinger. Ph.D. thesis. 1996. 
11. Meijman TF, Mulder, G. Psychological Aspects of Workload. In:

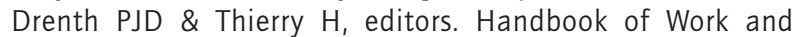
Organizational Psychology. Hove: Psychology Press. 1998;2:5-33.

12. Meijman TF, Schaufeli WB. Psychological fatigue and work. Psychologist. 1996;6:236-42.

13. Glass DC, Singer JE. Urban stress: experiments on noise and social stressors. New York: Academic Press. 1972.

14. Mohr G. Die Erfassung psychischer Befindensbeeinträchtigungen bei Industriearbeitern. Frankfurt: Lang. 1986.

15. Jansen NWH, Kant I, Van Den Brandt PA. Recovery in the working population: Description and associations with fatigue and psychological distress. Int J Behav Med. 2002;9:322-40.

16. Kompier MAJ. Arbeid en gezondheid van stadsbuschauffeurs. Delft: Eburon. Ph.D. thesis. 1988.

17. Van Dijk FJH, Van Dormolen M, Kompier MAJ, Meijman TF. Herwaardering model belasting-belastbaarheid [Revaluation of the model work load-work capacity]. Tijdschrift Sociale Gezondheidszorg [Journal Social Health Care]. 1990;68:3-10.

18. Jansen AWH, Kant I, Kristensen TS, Nijhuis FJN. Antecedents and consequences of work-family conflict: A prospective cohort study. J Occup Environ Med. 2003;45:479-91.

19. Meijman TF, Cavalini $P$, Creemer R, Van Dormolen M, Gloerich F, Hellinga $P$, et al. Onderzoek taakbelasting rijexaminatoren. Groningen: Department of Work Psychology, University of Groningen. 1985.

20. Broersen JPJ, Fortuin RJ, Dijkstra I, Van Veldhoven M, Prins J. Monitor Arboconvenanten: kengetallen en grenswaarden [Monitor Working Conditions Agreements: indicators and cut-offs]. Tijdschrift voor Bedrijfs- en Verzekerings-geneeskunde [Journal of occupational and insurance medicine]. 2004;12:100-4.

21. Sluiter JK, Van der Beek AJ, Frings-Dresen MHW. The influence of work characteristics on the need for recovery and experienced health: A study on coach drivers. Ergonomics. 1999;42:573-83.

22. Van Veldhoven $M, \&$ Broersen $S$. Measurement quality and validity of the "need for recovery scale". Occup Environ Med. 2003;60:3-9.

23. Jansen NWH, Kant I, Van den Brandt PA. Need for recovery in the working population: Description and associations with fatigue and psychological distress. Int J Behav. 2002;9:322-40.

24. Sluiter JK, de Croon EM, Meijmann TF, Frings-Dresen MHW. Need for recovery from work related fatigue and its role in the development and prediction of subjective health complaints. Occup Environ Med. 2003;60:62-70.

25. Kiss $P$, De Meester M. Need for recovery in ageing workers. Int Congr Ser. 2005;1280:202-7.

26. Sonnentag S, Aijlstra FRH. Job characteristics and off-job activities as predictors of need for recovery, well-being, and fatigue. J Appl Psychol. 2006:91:330-50.

27. Demerouti E, Taris TW, Bakker AB. Need for recovery, home-work interference and performance: is lack of concentration the link? J Vocat Behav. 2007;71:204-20.

28. Sluiter JK, Frings-Dresen MHW, Van der Beek AJ, Meijman TF. The relation between work-induced neuroendocrine reactivity and recovery, subjective need for recovery, and health status. J Psychosom Res. 2001;50:29-37.

29. Qi W, Liu Y, Zhang J, Ji S, Sluiter JK, Zhou R, et al. Relalationship between work strain, need for recovery after work and cumulative cortisol amoung kindergarten teachers. Int Arch Occup Environ Health. 2015;88:1053-9.

30. De Croon EM, Sluiter JK, Frings-Dresen MHW. Need for recovery after work predicts sickness absence. A 2-year prospective cohort study in truck drivers. J Psychosom Res. 2003;55:331-9.

31. De Croon EM, Sluiter JK, Blonk RWB, Broersen JPJ, FringsDresen MHW. Stressful work, psychological job strain, and turnover: A 2-year prospective cohort study of truck drivers. J Appl Psychol. 2004;89:442-54.

32. Van Amelsvoort I, Kant IJ, Bultmann U, Swaen GMH. Need for recovery after work and the subsequent risk of cardiovascular disease in a working population. Occup Environ Med. 2003;60:83-7.

33. Eriksen $H R$, Ihlebaek $C$, Jansen JP, Burdorf $A$. The relations between psychosocial factors at work and health status among workers in home care organizations. Int J Behav Med. 2006;13:183-92.

34. Maslach C, Jackson SE, Leiter MP. Maslach Burnout Inventory. Manual. $3^{\text {rd }}$ ed. Palo Alto, CA: Consulting Psychologists Press. 1996.

35. Pace F, Lo Cascio V, Civilleri A, Guzzo G, Foddai E, Van Veldhoven M. The Need for Recovery scale: adaptation to the Italian context. Eur Rev Appl Psychol. 2013;63:243-9.

36. De Vries J, Michielsen HJ, Van Heck GI. Assesment of fatigue among working people: a comparison of six questionnaires. Occ Env Med. 2003;60:i10-i15.

37. Schellekens JMH, Brouwer J, Meijman TF. Determinants and effect of work pressure in building and construction, wood industry and house rental institutions. Groningen: Department of Work Psychology, University of Groningen. 1999.

38. De Croon EM, Blonk RWK, Frings-Dresen MHW, Van Der Beek AJ. Stress in transport: final report. Amsterdam: Coronal Institute. 2000.

39. Schaufeli WB, Van Dierendonck D. UBOS - The Utrecht Burnout Scale. Manual. Lisse: Swets and Zeitlinger. 2000.

40. Sonnentag S, Fritz C. The recovery experience questionnaire: development and validation of a measure for assessing recuperation. J Occup Health Psych. 2007;12:204-21.

41. Maslach C., Leiter MP. The truth about burnout. San Francisco: Jossey Bass. 1997.

42. Boudoukha, AH. Burn-out et stress post-traumatique. Paris: Dunod. 2016.

43. Mohren DCL, Jansen NWH, Kant, IJ. Need for recovery from work in relation to age: a prospective cohort study. Arch Occup Environ Health. 2010;83:553-61.

44. Kiss P, De Meester M, Braeckman L. Differences between younger and older workers in the need for recovery after work. Int Arch Occup Environ Health. 2008:81:311-20.

45. Formanoy MAG, Dusseldorp E, Coffeng JK, Van Mechelen I, Boot CRL, Hendriksen IJM, Tak, ECPM. Physical activity and relaxation in the work setting to reduce the need for recovery: what works for whom? BMC Public Health. 2016;16:866. 